\title{
Public health primary prevention implemented by clinical high- risk services for psychosis
}

\author{
Andrés Estradé ${ }^{1}$, Gonzalo Salazar de Pablo $\mathbb{i D}^{1,2,3}$, Alice Zanotti ${ }^{4}$, Scott Wood ${ }^{5}$, Helen L. Fisher ${ }^{6,7}$ and Paolo Fusar-Poli (iD ${ }^{1,4,8,9 凶}$ \\ (c) The Author(s) 2022
}

Clinical High Risk for Psychosis (CHR-P) services have been primarily developed to support young people with attenuated symptoms (indicated prevention). No evidence-based appraisal has systematically investigated to what extent these clinics may implement other preventive approaches. PRISMA 2020-compliant systematic review of Web of Science, Cochrane Central Register of Reviews, and Ovid/PsychINFO, from inception until 14th June 2021, identifying original studies describing public health strategies: (a) service characteristics (configuration of mental health service, outreach, pathways to care); (b) universal interventions (general population); (c) selective interventions targeting CHR-P service-users or family/carers. Public health preventive initiatives were systematically stratified according to core social determinants of mental disorders associated with the 2030 Sustainable Development Goals promoted by the United Nations Member States (UN 2030 SDG) and good mental health outcomes. A total of 66 publications were included, providing data on 13 standalone, 40 integrated, three networks, and six regional or international surveys of CHR-P services across Europe, Asia, Oceania, Africa, North and South America, providing care to $>28 \mathrm{M}$ people. CHR-P services implement numerous public health initiatives targeting social and cultural (16 initiatives), economic (seven initiatives), demographic (six initiatives), environmental events (four initiatives) and neighbourhood (three initiatives) UN 2030 SGD determinants of mental disorders. There is additional evidence for CHR-P services promoting good mental health. The main barriers were the lack of resources for expanding public health prevention at a large scale. CHR-P services implement numerous public health prevention initiatives and promotion of good mental health beyond indicated prevention of psychosis.

Translational Psychiatry (2022)12:43; https://doi.org/10.1038/s41398-022-01805-4

\section{INTRODUCTION}

A recent large-scale meta-analysis across 192 epidemiological studies $(n=708,561)$ found that $48.4 \%$ of mental disorders have their onset before age 18, with an overall peak at 14.5 years [1]. Efficacy of treatments is limited after the onset of mental disorders, leading to a high long-term burden upon individuals, families, healthcare systems and society more broadly [2]. Prevention in young people, therefore, represents a promising avenue to improve outcomes of mental disorders [3].

Primary prevention strategies may target the general population (i.e. universal); subgroups of people at higher-than-average risk of developing mental disorders (i.e. selective); or individuals with emerging or subthreshold manifestations of mental disorders (i.e. indicated) [4]. The Clinical High Risk for Psychosis paradigm (CHR-P) [5] indicated preventive model includes help-seeking adolescents or young adults (typically 14-35 years [6]) who accumulate risk factors for psychosis [7], often concurrent with functional [8] and neurocognitive impairments [9] and mild or infrequent symptoms of psychosis [5]. CHR-P individuals have a $25 \%$ increased risk of developing psychosis over the following 3 years, which is about 50fold higher than in age-matched controls [10]. Specialised CHR-P services have been implemented in all six continents [11] to detect, formulate a prognosis (at risk or not at risk of transition to psychosis) and provide preventive care [6]. As an indicated preventive approach, CHR-P research has mostly focused on prevention of psychosis or other outcomes in young people presenting to these services. However, clinical care implemented by CHR-P services frequently encompasses public health initiatives that selectively target CHR-P individuals or their carers/family or universal interventions targeting the general population [12], as well as promotion of good mental health and wellbeing (as opposed to prevention of mental disorders [13]). Accordingly, CHR-P services may have the potential to address several potentially modifiable social determinants of psychosis risk, such as demographic factors (e.g. ethic related factors), economic factors (e.g. poverty), neighbourhoodrelated factors (e.g. social deprivation, infrastructure, urbanicity), exposure to traumatic environmental events (e.g. migration), violence (e.g. physical or emotional abuse) and natural/industrial

\footnotetext{
${ }^{1}$ Early Psychosis: Interventions and Clinical-detection (EPIC) Lab, Department of Psychosis Studies, Institute of Psychiatry, Psychology \& Neuroscience, King's College London, London, UK. ${ }^{2}$ Institute of Psychiatry and Mental Health, Department of Child and Adolescent Psychiatry, Hospital General Universitario Gregorio Marañón School of Medicine, Universidad Complutense, Instituto de Investigación Sanitaria Gregorio Marañón (liSGM), CIBERSAM, Madrid, Spain. ${ }^{3}$ Child and Adolescent Mental Health Services, South London and Maudsley NHS Foundation Trust, London, UK. ${ }^{4}$ Department of Brain and Behavioral Sciences, University of Pavia, Pavia, Italy. ${ }^{5}$ Yale School of Medicine, Yale University, New

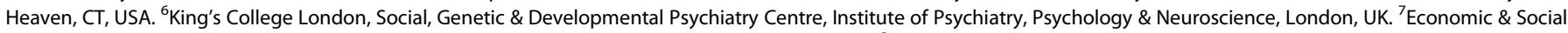

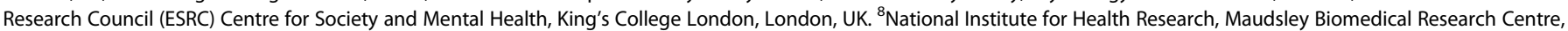
South London and Maudsley NHS Foundation Trust, London, UK. ${ }^{9}$ OASIS Service, South London and Maudsley NHS Foundation Trust, London, UK.

email: paolo.fusar-poli@kcl.ac.uk
}

Received: 24 August 2021 Revised: 8 January 2022 Accepted: 14 January 2022

Published online: 28 January 2022 
disasters, and social and cultural factors such as education, social support, and social/cultural capital [14].

This potential has never been systematically appraised, highlighting a profound gap of knowledge. The primary aim of this systematic review is to provide an evidence-based systematic appraisal of public health initiatives implemented by CHR-P services worldwide, in the context of an established public health framework identifying social determinants of mental disorders [14], and complemented by good mental health domains [13].

\section{METHODS}

This systematic review was pre-registered (study protocol: PROSPERO CRD42020163640) and conducted in accordance with the "preferred reporting items for systematic reviews and metaanalyses" (PRISMA 2020) [15] guidelines.

\section{Search strategy and selection criteria}

Three independent researchers ( $A E, G S d P$ and $A Z$ ) conducted the literature search and screening process for the identification of relevant articles. Discrepancies were resolved via consultation with a senior researcher (PFP). The search included the Web of Science database (Clarivate Analytics, incorporating the Web of Science Core Collection, BIOSIS Citation Index, KCI-Korean Journal Database, MEDLINE, Russian Science Citation Index, and SciELO Citation Index), Cochrane Central Register of Reviews, and Ovid/PsychINFO databases, from inception until 14th June 2021. The search terms used are appended in the eMethod 1. Titles and abstracts were screened, and potential full texts were assessed against inclusion and exclusion criteria. References of selected studies were screened for manual inclusion of any additional relevant publication.
Inclusion criteria were: (a) being an original study published in a scientific journal; (b) providing descriptive information of one or more CHR-P clinical services, as defined by established CHR-P assessment instruments (eMethod 2); and (c) providing information on different public health preventive interventions (defined as indicated below). Exclusion criteria were: (a) non-relevant designs such as secondary studies (i.e. reviews, meta-analyses, umbrella reviews), abstracts, conference proceedings, protocols, guidelines; (b) non-relevant populations, such as studies describing research other than CHR-P clinical services; and (c) no information on public health interventions. There was no restriction of language, and overlapping samples were not excluded.

\section{Measures and data extraction}

Descriptive information for CHR-P services was systematically extracted by two independent researchers, and discrepancies were resolved via consultation with a senior researcher. Extracted information included: (i) general service information: name of CHR-P service, region and country, CHR-P age inclusion criteria, catchment area population, type of service/data (standalone, integrated, networks or surveys) [12]; and (ii) type of public health initiatives implemented (see below).

\section{Data synthesis}

Public health initiatives were systematically clustered using a public health research framework focusing on core social determinants of mental disorders (Table 1). A large umbrella review addressed public health initiatives targeting social determinants of psychotic, bipolar and common mental disorders and empirically linked them with the 2030 Sustainable Development Goals (SDG) promoted by the United Nations Member States in 2015 [14] (UN 2030 SDG). Accordingly,

Table 1. Essential public health interventions for social determinants of mental disorders associated with the UN 2030 Sustainable Development Goals (SDG), adapted from Lund 2018 [14].

\section{Social determinants and description}

\section{Demographic}

Demographic characteristics of populations that convey risk for, or protection from, mental illness.

\section{Economic}

Factors related to the production, consumption, and transfer of wealth that convey risk for, or protection from, mental illness.

\section{Neighbourhood}

features of an area or community that convey an increased risk, or protection from, mental illness.

\section{Environmental events}

Serious events that disrupt a community's ability to cope and convey an increased risk for mental illness.

\section{Social and cultural}

Factors related to the organization of society, relationships, and social interactions that convey risk for, or protection from, mental illness.

\section{Relevant SDGs}

SDG 5: achieve gender equality and empower all women and girls.

SDG 1: end poverty in all forms; SDG 2: end hunger and achieve food security; SDG 8: promote decent and sustainable work and economic growth; SDG 9: build resilient industry, innovation, and infrastructure; SDG 10: reduced inequalities within and among countries.

SDG 6: ensure access to clean water and sanitation; SDG 7: ensure access to affordable and clean energy; SDG 11: make cities and communities sustainable and safe; SDG 12: ensure responsible consumption and production patterns.

SDG 13: take urgent action to combat climate change and its impacts; SDG 16: promote peace, justice, and strong institutions.

SDG 4: ensure inclusive and quality education for all.

\section{Distal and proximal socia determinants of mental disorders}

Distal: community diversity, population diversity, longevity, survival.

Proximal: age, ethnicity, gender.

Distal: economic recession, economic inequality, macroeconomic policy. Proximal: income, debt, assets, financial strain, relative deprivation, unemployment, food security.

Distal: infrastructure, neighbourhood deprivation, built environment, setting. Proximal: safety and security, housing structure, overcrowding, recreational facilities/opportunities.

Distal: natural disasters, industrial disasters, war or conflict, climate change, forced migration.

Proximal: trauma, distress.

Distal: community and social capital, social stability, cultural. Proximal: individual social capital, social participation social support, education.
Potential public health interventions

Reduction of gender-based violence, child maltreatment, and racial discrimination and xenophobia.

Cash transfers or basic income grants, reductions in income inequality, and improved employment.

Improved housing, safe neighbourhoods.

Reductions in violence, early response to environmental events, and action on protecting vulnerable ecosystems.

Improved education, strengthened social capital, and improving social support and networks for older adults. 
public health initiatives were clustered across the following SDG domains: (i) demographic, (ii) economic, (iii) neighbourhood, (iv) environmental events, and (v) social and cultural domains. Within each of these domains, public health interventions were further stratified into three subcategories pertaining to (a) service characteristics (e.g. configuration of the mental health service, outreach, pathways to care); (b) universal interventions targeting the general population at a country, regional, city or neighbourhood level; and (c) selective interventions targeted to CHR-P service-users or family/ carers but not primarily focusing on their presenting symptoms and risk for psychosis. As promotion of good mental health and wellbeing (as opposed to prevention, which is more concerned with avoiding mental disorders) is a core strategy of public health approaches, we additionally linked the public health interventions identified in the subcategories ( $b$ and $c$ ) to core good mental health domains. The latter were empirically validated in a previous consensus exercise (eTable 1): mental health literacy, attitudes towards mental disorders, self-perception and values, cognitive skills, academic/occupational performance, emotions, behaviours, self-management strategies, social skills, family and significant relationships, physical health, sexual health, meaning of life, and quality of life $[13,16]$.

\section{RESULTS}

\section{Study selection}

A total of 13,558 citations were screened for initial eligibility, and 318 full-text studies were evaluated. The final database consisted of 66 studies (Fig. 1). It included 13 standalone CHR-P services from Australia [17-20], Brazil [21], Germany [22], Netherlands [23], Poland $[24,25]$, South Korea [26], Singapore [27, 28], UK [29-36], and US $[37,38]$, and 40 integrated CHR-P services from Australia [39-42], Canada [43, 44], Chile [45], China (Hong Kong) [46-48], France [49], Greece [50, 51], Italy [52-61], Japan [62], Norway [63, 64], South Korea [65], Spain [66, 67], Switzerland [68-71], Tunisia [72], UK $[36,73,74]$; three networks of CHR-P services: the Early Detection Intervention and Prevention of Psychosis Program (EDIPPP) $[75,76]$, the Pan-London Network for Psychosis Prevention (PNP) [36], and Swiss Early Psychosis Project (SWEPP) [70]; five studies on national

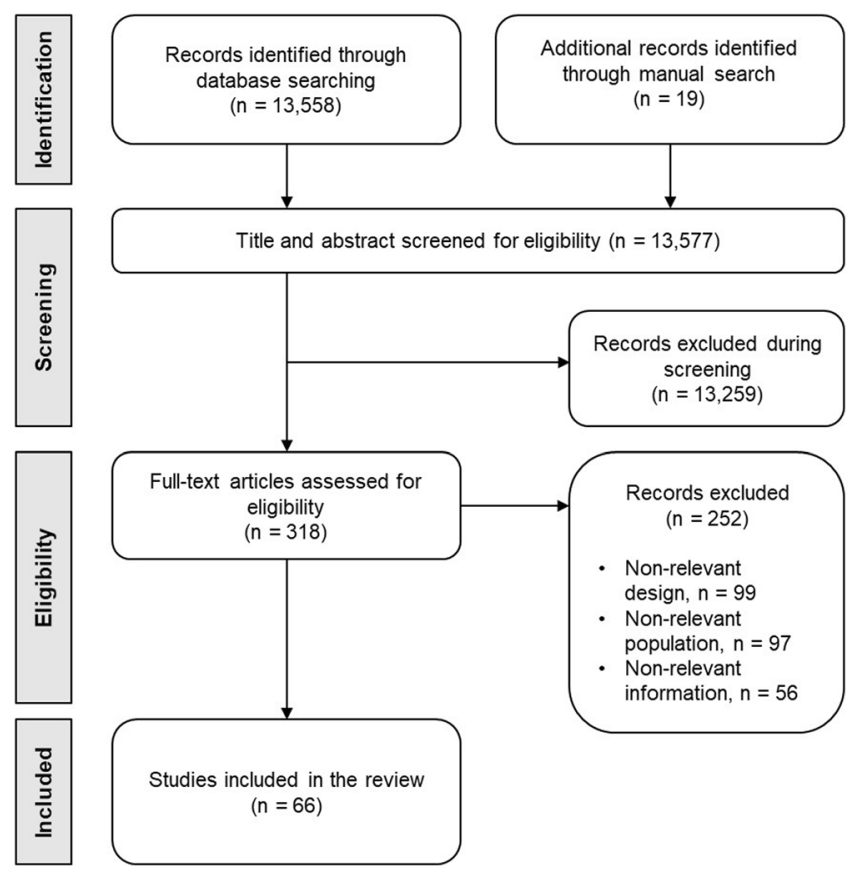

Fig. 1 Study selection flow-chart. Preferred reporting items for systematic reviews and meta-analyses (PRISMA) flowchart outlining study selection process. or regional surveys: 18 Early Intervention Services (EIS) from Canada [77], 45 [78] and 46 [79] EIS from Italy (two different surveys), 11 EIS from Portugal [80], and 50 EIS from the United Kingdom [81]; and one global survey of 47 CHR-P services [11] (eFig. 1). Overall, the CHR-P services included provided care to over $28 \mathrm{M}$ people. Full details of the studies included in this review and CHR-P services are available in Table 2 and eTables $2-4$.

\section{Demographic domain}

Demographic factors linked to an increased risk of mental disorders include distal factors, such as community and population diversity, longevity, and survival, and proximal factors, including age, gender, and ethnicity (Table 1) [14]. Related to this domain, the UN has postulated the achievement of gender equality and empowerment of all women by 2030 (SDG 5) [82]. These proximal factors were all targeted through CHR-P service characteristics (Table 3).

CHR-P services frequently adopted an anti-stigma and youthfriendly setting to increase the attractiveness of services and pathways to care for young people [17, 20, 35, 52, 65], typically opting for a community location far from main mental health hospitals or clinics and closer to public areas visited by young people $[44,53]$. CHR-P services also implemented a flexible and community outreach approach to enhance access of young people, facilitating encounters outside standard clinical settings [37] or offering case-management via smartphone applications [65].

Likewise, CHR-P services were often located in catchment areas with a high proportion of ethnic minorities [29, 30, 35, 76], or implemented community engagement projects specifically targeting young people from black, Asian and other minority groups [32]. CHR-P samples varied greatly in terms of ethnical composition, ranging from $>80 \%$ White in Italian [59-61] and Polish [25] services to non-White representing $>30 \%$ of clinical samples in US services $[37,75,76],>50 \%$ in the UK services $[29,31-34,36]$, and up to $80 \%$ in Canadian samples [77], demonstrating that the paradigm is successful in reaching ethnically diverse audiences.

CHR-P services involved current and past service-users in developing and improving service delivery to young people with diverse cultural backgrounds [27, 32, 36, 77-79]. These activities encompassed the development of information material sensitive to youth culture [27], the creation of servicespecific websites [36], the adaptation of service delivery to ethnically and culturally diverse populations [32], and the inclusion of peer support [77]. In terms of gender diversity, lesbian, gay, bisexual, transgender $+(\mathrm{LGBT}+)$ populations represented $25.9 \%$ of the clinical samples in one Australian service [17], where efforts to further enhance and adapt service provision to this population are currently underway.

\section{Economic domain}

Economic factors linked to increased risk of mental disorders range from macroeconomic, such as recession, inequality, and policy, to proximal factors including income, debt, assets, financial strain, relative deprivation, unemployment, and food security (Table 1) [14]. UN 2030 SDG linked to this domain include the eradication of poverty (SDG 1) and hunger (SDG 2), the promotion of sustainable growth and decent work conditions (SDG 8), resilient infrastructure, inclusive and sustainable industry, and innovation (SDG 9), and the reduction of inequalities (SDG 10) [82]. Economic factors were targeted by CHR-P via service characteristics and selective strategies (Table 3 ).

In terms of service characteristics, CHR-P services were at times located in catchment areas with high levels of unemployment, homelessness, and low socioeconomic status [29, 30, 35]. Lack of occupational activity among clinical samples in CHR-P services was not uncommon, ranging from $10-29 \%[25,62,76]$, to $30-40 \%$ $[29-33,39,50,55]$, and $>40 \%[36,45]$. In addition, a recoveryoriented service model was frequently adopted by CHR-P services 
Table 2. Description of CHR-P services included in the review.

\section{Service(s)}

\section{Standalone CHR-P services}

PACE [18-20]

CASPAR [17]

ASAS [21]

FETZ Cologne [22]

UMC EIS [23]

PORT $[24,25]$

Seoul Youth Clinic [26]

SWAP $[27,28]$

PHK EIS [70]

OASIS [29-36]

THEDS [36]

RAP $[75,76]$

PIER $[37,38,75,76]$

\section{Integrated CHR-P services}

PAS [40]

EIS [39]

SVH EPP [41, 42]

CAYR [43]

PRIME [44]

UCHIP [45]

EASY [46-48]

C'JAAD [49]

Eginition University Hospital EIS $[50,51]$

Catanzaro DMH EIS [52]

Grosseto DMH EIS [52]

Rome (area D) DMH EIS [52]

Salerno DMH EIS [52]

Programma 2000 [52, 56-58]

CCM2013 Project [53]

Ferrara DMH EIS [54]

Pr-EP [55]

ReARMS [59-61]

SAFE [62]

POP $[63,64]$

Mindlink [65]

ECEARP [66]

PAE-TPI [67]

FEPSY $[69,70]$

EIS [70]

FETZ Bern [70, 71]

JADE [70]

TIPP [70]

Station FP [70]

FES [70]

ZInEP Study $[68,70]$

CHiRP [72]

EIS [73]

HEADS UP [36]

NEIS [36]

$$
\text { Country - Region/City }
$$

Australia-Melbourne

Australia-Sydney

Brazil-São Paulo

Germany-Cologne

Netherlands-Utrecht

Poland-Lodz

South Korea-Seoul

Singapore

Switzerland-Aargau

UK-London

UK-London

US-New York

US-Portland

Australia-Newcastle

Australia-New South Wales

Australia-Melbourne

Canada-Montreal

Canada-Toronto

Chile-Santiago

China-Hong Kong

France-Paris

Greece-Athens

Italy-Catanzaro

Italy-Grosseto

Italy - Rome

Italy - Salerno

Italy-Milan

Italy-Lombardy, Tuscany and Liguria

Italy-Ferrara

Italy-Parma

Italy-Reggio Emilia

Japan-Sendai

Norway-Stavanger

South Korea-Gwangiu

Spain-Barcelona

Spain-Catalonia

Switzerland-Basel

Switzerland-Basel-Bruderholz

Switzerland-Bern

Switzerland-Geneva

Switzerland-Lausanne

Switzerland-Münsterlingen

Switzerland-Winterhur

Switzerland-Zurich

Tunisia-Tunis

UK-Lincolnshire

UK-London

UK-London
CHR-P age inclusion*

14-30 years

12-25 years

14-30 years

16-40 years

12-25 years

14-29 years

15-35 years

16-30 years

NA

14-35 years

16-35 years

NA

12-35 years

NA

12-24 years

16-65 years

14-35 years

14-30 years

12-35 years

12-25 years

$15-30$ years

$15-40$ years

17-30 years

17-30 years

17-30 years

17-30 years

17-30 years

15-24 years

15-35 years

12-35 years

13-35 years

14-35 years

13-65 years

$15-30$ years

12-56 years

18-35 years

$>18$ years

NA

8-40 years

18-25 years

18-35 years

16-25 years

16-35 years

13-35 years

NA

14-65 years

18-35 years

18-35 years
Catchment area population

NA

NA

NA

$1,000,000$

NA

$1,000,000$

NA

NA

NA

$1,358,646$

317,203

557,725

333,000

NA

NA

245,000

$1,900,000$

2,500,000

NA

$7,000,000$

NA

NA

35,000

65,000

250,000

100,000

200,000

NA

360,000

500,000

550,000

$1,060,000$

300,000

$1,500,000$

83,567

NA

200,000

NA

$1,000,000$

NA

250,000

NA

NA

$1,300,000$

NA

750,000

281,740

353,245 
Table 2. continued

\begin{tabular}{l} 
Service(s) \\
\hline Teesside EIP Service [74] \\
\hline EDAPT $[75,76]$ \\
M3P $[75,76]$ \\
EARLY $[75,76]$ \\
EAST $[75,76]$ \\
Surveys of EIS
\end{tabular}

\section{Survey of 47 CHR-P}

services [11]

Survey of 18 EIS [77]

Survey of 45 EIS [78]

Survey of 46 EIS [79]

Survey of 11 EIS [80]

Survey of 50 EIS [81]

\section{Country - Region/City}

UK-Teesside

US-California

US-Michigan

US-New Mexico

US-Oregon

International-Africa, Asia, Europe, North America,

South America, Oceania

Canada-Quebec

Italy-Nationwide

Italy-Nationwide

Portugal-Oporto, Santa Maria da Feira, Coímbra,

Caldas da Rainha, Leiria, Lisbon, Faro

UK-England

\begin{tabular}{ll}
$\begin{array}{l}\text { CHR-P age } \\
\text { inclusion* }\end{array}$ & $\begin{array}{l}\text { Catchment area } \\
\text { population }\end{array}$ \\
\hline $14-35$ years & 600,000 \\
\hline NA & 466,488 \\
NA & 344,791 \\
NA & 661,422 \\
NA & 631,853 \\
\hline NA & NA \\
\hline NA & $\approx 3,750,000$ \\
\hline NA & $\approx 200,000$ to 400,000 \\
\hline NA & NA \\
\hline NA & $3,400,000$ \\
\hline $14-35$ (85\%) & NA \\
\hline
\end{tabular}

ASAS Evaluation and Follow-up of Adolescents and Young Adults in São Paulo, CASPAR Comprehensive Assessment Service for Psychosis and At Risk, CAYR Clinic for Assessment of Youth at Risk, CCM National Centre for Disease Prevention and Control, CHiRP clinical high-risk program of Razi Hospital, CHR-P clinical high-risk of psychosis, C'JAAD Evaluation Centre for Adolescents and Young Adults, DMH Department of Mental Health, EARLY Early Assessment and Resource Linkage for Youth, ECEARP Care Equipment for At-Risk of Psychosis Patients, EAST Early Assessment and Support Team, EASY Early Assessment Service for Young people with psychosis, EDAPT Early Detection and Preventive Treatment, EIP early intervention in psychosis, EIS early intervention service, FEPSY Basel early-detection-of-psychosis study, FETZ Early Recognition and Intervention Centre for Mental Crises, HEADS UP City \& Hackney At-Risk Mental State Service, M3P Michigan Prevents Prodromal Progression, NA not available or unclear data, NEIS Newham Early Intervention Service, OASIS Outreach and Support in South London, PACE Personal Assessment and Crisis Evaluation clinic, PAE-TPI Early Psychotic Disorder Care Programmes, PAS Psychological Assistance Service, PHK Psychiatric Hospital Königsfelden, PIER Portland Identification and Early Referral, POP Prevention of Psychosis study, PORT Programme of Recognition and Therapy, Pr-EP Parma-Early Psychosis programme, PRIME, Toronto Prevention through Risk Identification, Management and Education, RAP Recognition and Prevention programme, ReARMS Reggio Emilia At-Risk Mental States programme, SAFE Sendai ARMS and first episode clinic, Station FP Psychiatric Hospital Münsterlinger Early Psychosis Outpatient Service, SVH EPP St Vincent's Hospital early psychosis programme, SWAP Support for Wellness Achievement Programme, THEDS Tower Hamlets Early Intervention Service, TIPP Treatment and early Intervention in Psychosis Programme, UCHIP University of Chile Highrisk Intervention Program, UK United Kingdom, UMC University Medical Centre, US United States, ZInEP Zurich Early Recognition Program. *General age inclusion criteria are reported if CHR-P-specific data is not available.

through the enhancement of social and academic/occupational functioning among young users $[17,27,28,30,36,51,55,59-$ $61,63,64]$.

With respect to selective interventions, CHR-P services assessed vocational history, goals and engagement, and cognitive functioning as part of their standard operations [34, 71, 76]. Services also provided occupational or supportive therapy on vocational/ occupational functioning, with interventions such as group activities (e.g. computer training), support job seeking and retention [11, 34, 36, 42-44, 50-52, 55, 57, 58, 77-79]. In addition, CHR-P services provided specific programmes for onsite vocational reintegration, such as supported employment or individual placement and support (IPS) [34, 35, 54, 55, 73, 76, 80], often through intense networking with local/community stakeholders $[19,28,34,38,41,74,77]$.

Additional support extended to housing and accommodation via practical advice or coordination with housing services $[11,19,33,74,77]$. Homelessness among clinical samples of CHR-P services was reported at $3.0 \%$ in a UK service [32], and supported accommodation ranged from $3.3 \%$ [17] up to $17.5 \%$ [32]. Across different countries, such as Australia [17], France $[49,77]$, Japan [62], the UK [32], and Poland [25], most users live with their family or relatives, with only a minority living independently.

Through these initiatives, CHR-P services can impact academic/ occupational performance, cognitive skills and quality of life and promote good mental health.

\section{Neighbourhood domain}

Neighbourhood-related distal factors linked to an increased risk of mental illnesses include infrastructure, neighbourhood deprivation, built environment, and setting, and proximal factors include safety and security, housing structure, overcrowding, and access to recreational opportunities or facilities (Table 1) [14]. UN 2030 SDG include clean water and sanitation (SDG 6), access to affordable and reliable energy (SDG 7), sustainable, safe, and inclusive cities (SDG 11 ), and sustainable production and consumption (SDG 12) [82]. Neighbourhood-related factors have been targeted by CHR-P via service characteristics and selective strategies (Table 3 ).

In terms of service characteristics, CHR-P services have been located in neighbourhoods with high levels of social deprivation, including robbery, assault, substance use, and single people [30, 35, 74]. The proportion of single/unmarried users was most frequently in the ranges of $>90 \%[25,28,45,49,52,56,57,59,61,62,76]$ or $80-90 \%$ [29-31, 33, 41], with levels of users without a steady relationship or partner in the range of $80-92 \%[22,32,36]$ (eTable 3). However, most studies do not discriminate between marital and interpersonal relationship status (i.e. with or without a steady relationship). In addition, the proportion of married CHR-P users appears generally lower than the national averages for the corresponding age categories (eTable 3), although these data have not been subjected to formal statistical comparison. This domain is also targeted by the presence of an assessment and detection team in prison for one UK service [30].

Selective strategies include recreational opportunities-related interventions, encompassing recreational therapy [77], social group activities (e.g. music, multimedia, expression) $[52,57,58,77]$, support in planning recreational activities [58], and engaging with different community organisations [53].

Through these activities, CHR-P services can impact the quality of life, family and significant relationships and promote good mental health. 
Table 3. Public health strategies delivered by CHR-P services targeting the social determinants of mental disorders, stratified by service characteristics, universal strategies, selective strategies, with the corresponding good mental health domains.

\section{Domains}

Demographic

Economic

Neighbourhood

\section{Environmental} events

Social and cultural

\section{Interventions}

\section{Service characteristics}

- Anti-stigma and youth-friendly community setting and service delivery. $[17,20,35,44,52,53,65]$

- Flexible outreach approach to enhance engagement of young people. [37, 65]

- Presence in catchment areas with high levels of ethnic minorities. [29, 30, 35, 76]

- Community engagement projects focused on young people from ethnic minorities. [32]

- Service-user involvement in service development and delivery to include multicultural backgrounds. [27, 32, 36, 77-79]

- Inclusiveness of LGBT+populations. [17]

\section{Service characteristics}

- Presence in catchment areas with high levels of economic inequality, unemployment, and homelessness. [29, 30, 35]

- Recovery-oriented model of service delivery with focus on social and role functional support. [17, 27, 28, 30, 36, 51, 55, 59$61,63,64]$

\section{Selective strategies and corresponding good mental health domains}

- Assessment of vocational history, goals and engagement, and cognitive functioning. $[34,71,76]$

- Occupational or supportive therapy on vocational/occupational functioning. [11, 34, 36, $42-$ $44,50-52,55,57,58,77-79]$

- Onsite vocational reintegration, including supported employment or IPS. $[34,35,54,55,73,76,80]$

-Intensive networking with local/community stakeholders. [19, 28, 34, 38, 41, 74, 77]

- Psychosocial support with housing and accommodation. [11, 19, 33, 74, 77]

\section{Service characteristics}

- Presence in catchment areas with high levels of social deprivation. [30, 35, 74]

- Presence in a prison setting. [30]

\section{Selective strategies and corresponding good mental health domains}

- Recreational therapy, activities or support. [43, 52, 53, 57, 58, 77]

Academic/occupational

\section{Service characteristics}

- Presence in catchment areas with high levels of refugees and asylum seekers. [29]

- Community engagement projects focused on asylum seekers and refugees. [32]

- Trauma-sensitive model of care. [39]

- Comprehensive assessment of lifetime exposure to traumatic events. [30, 67, 71]

\section{Universal strategies and corresponding good mental health domains}

- Mental health awareness and promotion campaigns for the general population, parents, and families. $[19,21,22,24,25,27,29,32,36-38,40,44-49,52,56,57,62-64,68-$ $70,75,77,79]$

- Service-user involvement for the promotion of mental health literacy. [32, 36]

- Psychoeducation groups in collaboration with community organisations. [77]

- Education, awareness and anti-stigma campaigns for community organisations. $[19,20,22,27,29,30,32,36-38,40,43,44,53,56,57,63,66,77-79]$

- Training and mental health awareness for professionals working with young people. $[19,20,22,24,27,29,32,35-38,40,44,45,49,50,52,54,56,57,62-64,68-$ $70,72,73,75,77-79]$

\section{Selective strategies and corresponding good mental health domains}

- Problem-solving training. $[18,57,58,78,79]$

- Individual or group psychoeducation. [18, 28, 31, 36, 49, 50, 58, 65, 71, 72, 74, 78-81]

- Life/practical skills training. [74, 77]

- Family psychoeducation, counselling or support. $[11,17,23,26,27,32,35,36,42,43,45,49,51,52,54-65,71-73,76-78,81]$

QoL, family and significant relationships

Academic/occupational performance, cognitive skills

Academic/occupational performance

Academic/occupational performance performance

QoL

Mental health literacy, attitude towards mental disorders

Mental health literacy, attitude towards mental disorders

Mental health literacy, attitude towards mental disorders

Mental health literacy, attitude towards mental disorders

Mental health literacy, attitude towards mental disorders

Self-management skills

Mental health literacy, selfmanagement skills

Self-management skills

Family and significant relationships 
Table 3. continued

Domains

\section{Interventions}

- Psychosocial support on social relationships and functioning.

$[11,27,28,30,36,43,45,49,50,54,55,58,59,77]$

- Educational support. $[11,27,28,35,36,49,52,57,58,74,76,77]$

- Cognitive Remediation Training [72]

- Physical health assessment and monitoring. [36, 39, 41, 42, 45, 73, 80]

- Exercise/physical activity intervention, psychomotor therapy. [17, 23, 39, 43, 65, 77-79]

- Nutrition and healthy eating intervention or advice. [39, 43, 49, 65, 74, 77]

- Sleep hygiene or sleep interventions. [18, 30]
Social skills

Academic/occupational performance

Cognitive skills

Physical health, QoL

Physical health, QoL

Physical health, QoL

Physical health, QoL

IPS individual placement and support, LGBT+ lesbian, gay, bisexual, transgender+, QoL quality of life.

\section{Environmental events domain}

Exposures to natural and industrial disasters, war or conflict, forced immigration, and ecosystems hazards (e.g. floods, droughts) produced by climate change have been reported as distal risk factors of mental disorders, alongside the more proximal factors of trauma and distress (Table 1) [14]. The UN 2030 SDG postulate the need for urgent action to tackle climate change and its impacts (SDG 13) and the promotion of peace, justice, and strong institutions (SDG 16) [82]. CHR-P services addressed some of these environmental events by their service characteristics (Table 3).

Regarding conflict, war, and migration, a CHR-P service in the UK reported being located in a catchment area with a high proportion of refugees and asylum seekers [29]. The proportion of immigrants or non-native speakers among service users ranged from $\approx 10-20 \%$ in European and Australian services $[17,22,39,49,55,60,66]$ to up to $80 \%$ in Canadian services [77]. Service delivery has been adapted to respond to traumatic exposures in different ways, either by conducting community engagement projects specifically targeting asylum seekers and refugees [32], or by adopting a trauma-sensitive model of care [39], and conducting comprehensive assessments of lifetime exposure to adverse events (e.g. sexual abuse, emotional abuse, physical abuse, and neglect) as part of regular clinical operations $[30,67,71]$.

\section{Social and cultural domain}

Social and cultural determinants of mental disorders encompass the distal factors of community, social and cultural capital, and social stability, and the proximal factors of individual social capital, participation and support, and education (Table 1) [14]. UN 2030 SDG linked to this domain include ensuring inclusive and equitable education for all (SDG 4) [82]. This domain was addressed by CHR-P services through universal or selective interventions (Table 3).

Within universal approaches, mental health awareness campaigns targeting the general population were commonly conducted at a community or city-wide level with the aim of improving mental health literacy, and reducing the stigma attached to mental disorders [19, 21, 22, 24, 25, 27, 29, 32, 36$38,40,44-49,52,56,57,62-64,68-70,75,77,79]$. Activities varied greatly, including workshops, presentations, talks, tv or radio appearences $[22,27,37,44,46-48,68]$, participation in public events, fairs and exhibitions [27, 38, 40, 44, 46-48, 75], articles and publications in local newspapers and other printed or audio-visual media (e.g. videos, leaflets, brochures) $[21,22,27,37,44,46-$ $48,62-64,69]$, and the use of online resources (e.g. service-specific website, social media) $[29,32,36,37,46-48,63,64,69,70]$. In Hong Kong, a new definition of "psychosis" was tested to improve a non-stigmatising engagement of the public [46-48]. On occasion, activities were conducted for targeted audiences, such as schools students, adolescents, or parents [24, 46-48, 57]. Other public awareness and education campaigns focused on specific community organisations or services $[19,20,22,27,29,30,32,36-$ $38,40,43,44,53,56,57,63,66,77-79]$, such as the development of a community preventive alliance alongside education, religious and other organisations [53]. Frequent community and public organisations targets encompassed: community healthcare services $[20,22,29,30,32,37,40,43,44,53,56,57,66,78,79]$, social $[32,38,56,66]$, housing and employment [32] services, schools and colleges [20,32, 37, 43, 44, 53, 57, 66, 78, 79], sports organisations [27], religious or faith centres [32, 37, 44, 53], multicultural groups [38, 53], charities and non-governmental organisations [29, 30, 32], wellbeing and youth centres $[27,32,38,40,44,57,66]$, family services [27, 37], welfare organisations [27], local shops [32], libraries [44], governmental services [27], justice institutions [66], police and armed forces $[27,37,44,56]$, and correction facilities [37]. CHR-P services often collaborated with service users for the promotion of mental health literacy, for example, through the development of online resources based on their lived experiences $[32,36]$. Psychoeducational groups in collaboration with community organisations (i.e. not restricted to patients) have also been employed [77].

Another universal approach implemented mental health awareness and training activities targeting professionals working in close contact with young people, commonly including GPs, mental health professionals, counsellors, private practitioners, teachers and educators, social workers, and front-line youth workers $[19,20,22,24,27,29,32,35-38,40,44,45,49,50,52,54,56,57,62-$ $64,68-70,72,73,75,77-79]$. These activities addressed issues such as stigma reduction, risk factors for mental disorders, best preventive practices, case recognition, and were delivered through workshops $[22,24,44,68]$, education sessions and informal meetings $[24,32,37,38,40,56,73,75,77]$, articles in professional journals and other printed media $[19,22,37,40,62,68]$, phone or email consultation [36, 62, 75], and websites specifically tailored to professionals [37]. Formal education courses for healthcare professionals, students and professionals from related areas were also implemented [19, 24, 27, 45, 49, 50, 70, 75, 77].

In terms of selective interventions, CHR-P services offered group problem-solving training $[18,57,58,78,79]$, individual or group psychoeducation $[18,28,31,36,49,50,58,65,71,72,74,78-81]$, and life/practical skills training $[74,77]$. Other initiatives focused on the familial and social network such as family psychoeducation, counselling and support, extensively provided by CHR-P services $[11,17,23,26,27,32,35,36,42,43,45,49,51,52,54-65,71-$ 73, 76-78, 81]. Social functioning and relationships were promoted via psychosocial support, which included supportive counselling, case management for social functioning, and social group activities (e.g. social skills training, empowerment activities) $[11,27,28,30,36,43,45,49,50,54,55,58,59,77]$. Other selective interventions promoting this good mental health domain included educational support $[36,52,57,58,77]$, encompassing: case management aimed at educational functioning [11, 27, 28], 
collaboration with community organisations for optimising school reintegration and coordination [35, 49], facilitation of the Individualised Education Plans [76], and the identification of individualised educational programmes [74]. The cognitive skills domain was addresses via selective Cognitive Remediation Training interventions not primarily focusing on psychotic symptoms [72]. Finally, several CHR-P services implemented selective interventions to improve physical health and quality of life, such as routine physical health assessment and monitoring $[36,39,41,42,45,73,80]$, exercise/physical activity intervention or psychomotor therapy $[17,23,39,43,65,77-79]$, nutrition and healthy eating intervention or advice $[39,43,49,65,74,77]$, and sleep hygiene/disturbances interventions $[18,30]$.

Through these activities, CHR-P services can impact mental health literacy, attitude towards mental disorders, selfmanagement skills, family and significant relationships, social skills, academic/occupational performance, cognitive skills, physical health and quality of life, and promote good mental health.

\section{DISCUSSION}

This is the first systematic review to systematically appraise the evidence for public health interventions implemented by CHR-P services.

CHR-P services included in this review encompassed Europe, Asia, Oceania, Africa, and North and South America, providing care to over $28 \mathrm{M}$ people in their respective catchment areas. CHR-P services were found to implement a wide range of public health approaches beyond indicated prevention through service characteristics, selective or universal interventions. The public health initiatives implemented by CHR-P services addressed core social determinants of mental disorders that align to the UN 2030 SDG [82], albeit to a variable degree. Public health initiatives implemented by CHR-P services targeted (in decreasing order of public health initiatives) social and cultural (16 initiatives), economic (7 initiatives), demographic (6 initiatives), environmental events ( 4 initiatives) and neighbourhood (3 initiatives) SGD determinants of mental disorders.

While the characteristics of these interventions have already been addressed above, it is important to highlight their close association with good mental health outcomes. Universal preventive interventions implemented by CHR-P services were strongly aimed at promoting mental health literacy and a positive attitude towards mental disorders, core dimensions of positive mental health [13], which are inversely related to stigma. Public stigma about mental disorders can lead to reduced help-seeking behaviour in young people and barriers to healthcare [83], reduced social networks, loneliness, fewer employment and housing opportunities, and an overall deterioration of mental state [84]. Accordingly, poor mental health literacy among the general population has been identified as a major barrier to the delivery of effective preventive interventions [26, 78, 79]. A recent meta-analysis found that psychoeducation through awareness and outreach campaigns in the general population is particularly effective to improve mental health literacy $(E S=0.69)$ [85]. Psychoeducation can help to disconfirm negative stereotypical beliefs about people with mental disorders and lead to positive attitudinal changes in the public, reducing stigma [86]. While psychoeducation represents a fundamental component of the needs-based intervention for CHR-P individuals [12], we demonstrate that CHR-P services extend its benefits to the general population, parents, families, community organisations and professionals working with young people, largely as part of their outreach campaigns. Notably, CHR-P service-users are frequently involved in customising these activities to the regional culture and sensitivity of young people [27, 32].

Selective interventions were mostly employed by CHR-P services to target the good mental health domains of self- management skills, social skills, family and significant relationships, academic/occupational performance, cognitive skills and overall quality of life. There is recent meta-analytic evidence supporting the efficacy of selective interventions for the promotion of quality of life ( $E S=0.46)$, social skills $(E S=0.37)$, academic and occupational performance $(E S=0.21)$ in young people [85]. Physical health also represents a core good mental health domain addressed via a variety of selective interventions fostering healthy eating habits and physical activity, physical health monitoring, and sleep interventions. Improving physical health is of pressing urgency not only in early psychosis [39, 87, 88], but in several other mental disorders [89] and in the young general population [90]. Meta-analytic evidence supports the efficacy of selective (and universal) interventions for the promotion of good physical health among young people $(E S=0.285)$, with physical therapy, relaxation, and exercise being the most effective interventions [85]. Improving physical health is a tantalizing public health strategy, making sense for concurrently reducing the risk of many other mental disorders [91] such as psychotic, bipolar and depressive/ anxiety disorders [92]. Strategically, the numerator of preventive cost and risk can be offset by a denominator of multiple preventable psychiatric and physical disease outcomes [93].

Taken together, the evidence reviewed indicates that CHR-P services are already implementing extensive public health preventive approaches beyond indicated intervention. This review supports the importance of increasing the roll out of these services across the globe and improved funding given the broader value and potential wider impact on population mental health of these services. At the same time, this review undertones the "prevention paradox" [94] argument, stating that as CHR-P services can only benefit a small minority of young people, they should be dismantled [95]. Indicated approaches are expected a priori to target the tip of the iceberg of the population-level risk and are thus complementary and not antithetical (as claimed [96]) to selective and universal approaches [4]. Future research and clinical practice should better integrate universal, selective and indicated approaches to synergistically and complementarily maximize their efficiency in young people [4]. A first step could be to overcome current barriers to mainstreaming public health interventions conducted by CHR-P services. The main barrier is the limited financial and political support for preventive services $[21,50,52,77,81]$ linked to the lack of a preventive culture of mental health systems in some countries [78, 79]. A recent international survey of 47 CHR-P services indicated that $51.1 \%$ identified lack of financial support and $42.6 \%$ inadequate staffing resources as key implementation barriers [11]. For example, occupational and social workers, which are pivotal for public health initiatives, are present in less than half of CHR-P services [12], and even well-established CHR-P services find it hard to deliver comprehensive occupational interventions [34]. These challenges often translated into the difficulty of extending public health prevention to more isolated and rural areas [77]. In countries where sufficient funding has been made available, like Australia, youth-friendly preventive mental health services have been implemented at scale [97]. Refined CHR-P services, which broaden their remit to support young people experiencing a wide range of sub-clinical mental health symptoms (e.g. bipolar and depression beyond psychosis) and have public mental health and promotion remits, could become a model for providing preventive care in the general population [98]. A further barrier is that CHR-P public health research has been hindered by the lack of a unitary empirical framework. This study demonstrated that it is possible to adapt the public health framework [14] linked to the UN 2030 SDG [82] to CHR-P research. Our attempt provides a heuristic platform to facilitate further public health research in the CHR-P arena. Future research should specifically refine outreach approaches combining extensive community education within ethnically diverse communities and the creation of collaborative networks 
$[32,75,99]$. Additional work is also needed to customise effective public health prevention to LGBT+ populations [17] and culturally diverse and migrant populations $[22,45,75]$. Expanding initiatives to promote good mental health through anti-stigma campaigns [75], vocational and academic rehabilitation, and exercise and physical health $[34,45]$ is also a relevant point on the research agenda.

The main limitation of this review is that information on public health initiatives in CHR-P services is scattered and fragmented, preventing the feasibility of meta-analysis. Few studies [75] addressed public health initiatives upfront within their primary aims. Furthermore, some relevant data such as type of funding to CHR-P services were generally not reported in the original papers. In addition, conclusions are limited by the scarcity of standardised reports on the effectiveness of universal and selective strategies of CHR-P services for promoting good mental health domains. While the efficacy of these strategies has been systematically appraised in recent meta-analyses by our group $[85,100]$, there is no robust effectiveness evidence relating to CHR-P services. This may be due to the fact that research in this area is still emerging. We hope that the current systematic review, by providing an empirical classification of these initiatives will foster future research in this area. A further limitation is that there was minimal data available from low- and middle-income countries (studies from LMICs may be better represented in databases such as Lilacs, African Journals Online, and Global Health) and from countries where CHR-P services had more recently been introduced (e.g. Brazil, Tunisia, India, or Sweden) [11]. The level of research and evidence in LMICs is still modest and should become a mainstream topic on the future research agenda. In Latin America, El services are in their early stages of development and mostly focused on FEP rather than CHR-P users [101, 102]

\section{CONCLUSION}

CHR-P services implement a wide range of public health prevention initiatives and interventions for the promotion of good mental health beyond indicated prevention of psychosis. These initiatives address empirically validated social determinants of mental disorders, which align with the UN 2030 SDG.

\section{REFERENCES}

1. Solmi M, Radua J, Miriam O, Croce E, Soardo L, Salazar De Pablo G, et al. Age at onset of mental disorders worldwide: a large-scale meta-analysis of epidemiological studies. Mol Psychiatry 2021. https://doi.org/10.1038/s41380-021-01161-7.

2. Fusar-Poli P, McGorry PD, Kane JM. Improving outcomes of first-episode psychosis: an overview. World Psychiatry. 2017;16:251-65.

3. World Health Organization. Prevention of mental disorders. Effective interventions and policy options. 2004. https://apps.who.int/iris/handle/10665/43027. 24 August 2021.

4. Fusar-Poli P, Correll CCU, Arango C, Berk M, Patel V, loannidis JJPA. Preventive psychiatry: a blueprint for improving the mental health of young people. World Psychiatry. 2021;20:200-21.

5. Fusar-Poli $P$, Borgwardt $S$, Bechdolf A, Addington J, Riecher-Rössler A, SchultzeLutter $F$, et al. The psychosis high-risk state: a comprehensive state-of-the-art review. JAMA Psychiatry. 2013;70:107-20.

6. Fusar-Poli P, Salazar De Pablo G, Correll CU, Meyer-Lindenberg A, Millan MJ, Borgwardt $S$, et al. Prevention of psychosis: advances in detection, prognosis, and intervention. JAMA Psychiatry. 2020;77:755-65.

7. Fusar-Poli P, Tantardini M, De Simone S, Ramella-Cravaro V, Oliver D, Kingdom J, et al. Deconstructing vulnerability for psychosis: meta-analysis of environmental risk factors for psychosis in subjects at ultra high-risk. Eur Psychiatry. 2017;40:65-75.

8. Fusar-Poli P, Rocchetti M, Sardella A, Avila A, Brandizzi M, Caverzasi E, et al. Disorder, not just state of risk: meta-analysis of functioning and quality of life in people at high risk of psychosis. Br J Psychiatry. 2015;207:198-206.

9. Catalan A, Salazar de Pablo G, Aymerich C, Damiani S, Sordi V, Radua J, et al. Neurocognitive functioning in individuals at clinical high risk for psychosis: systematic review and meta-analysis. JAMA Psychiatry 2021. https://doi.org/ 10.1001/jamapsychiatry.
10. Gonzalo SDP, Radua J, Pereira J, Bonoldi I, Arienti V, Besana F, et al. Probability of transition to psychosis in individuals at clinical high risk: an updated metaanalysis. JAMA Psychiatry. 2021;78:970-8.

11. Kotlicka-Antczak M, Podgórski M, Oliver D, Maric NP, Valmaggia L, Fusar-Poli P. Worldwide implementation of clinical services for the prevention of psychosis: the IEPA early intervention in mental health survey. Early Inter Psychiatry. 2020;14:741-50.

12. Salazar de Pablo G, Estradé $A$, Cutroni $M$, Andlauer O, Fusar-Poli P. Establishing a clinical service to prevent psychosis: what, how and when? Systematic review. Transl Psychiatry. 2021;11:43.

13. Fusar-Poli $P$, Salazar de Pablo G, De Micheli A, Nieman DH, Correll CU, Kessing LV, et al. What is good mental health? A scoping review. Eur Neuropsychopharmacol. 2020;31:33-46.

14. Lund C, Brooke-Sumner C, Baingana F, Baron EC, Breuer E, Chandra P, et al. Social determinants of mental disorders and the Sustainable Development Goals: a systematic review of reviews. Lancet Psychiatry. 2018;5:357-69.

15. Page MJ, McKenzie JE, Bossuyt PM, Boutron I, Hoffmann TC, Mulrow CD, et al. The PRISMA 2020 statement: an updated guideline for reporting systematic reviews. BMJ 2021; 372. https://doi.org/10.1136/bmj.n71.

16. Salazar De Pablo G, Catalan A, Fusar-Poli P. Clinical validity of DSM-5 Attenuated Psychosis Syndrome: advances in diagnosis, prognosis, and treatment. JAMA Psychiatry. 2020;77:311-20.

17. Yang R, Curtis J, Jensen C, Levy P, Chown K, Lappin JM. Detection and intervention in emerging youth mental health issues: outcomes from the first year of the CASPAR service. Early Interv Psychiatry. 2020;15:167-73.

18. Formica MJC, Phillips LJ, Hartmann JA, Yung AR, Wood SJ, Lin A, et al. Has improved treatment contributed to the declining rate of transition to psychosis in ultra-high-risk cohorts? Schizophr Res 2020. https://doi.org/10.1016/j. schres.2020.04.028.

19. Phillips L, Leicester S, O'Dwyer L, Francey S, Koutsogiannis J, Abdel-Baki A, et al. The PACE Clinic: identification and management of young people at "ultra" high risk of psychosis. J Psychiatr Pr. 2002;8:255-69.

20. Yung AR, Phillips LJ, McGorry PD, Hallgren MA, McFarlane CA, Jackson $H J$, et al. Can we predict the onst of first-episode psychosis in a high-risk group? Int Clin Psychopharmacol. 1998;13:S23-30.

21. Louza MR, Azevedo Y, Macedo G, Gattaz W. An early psychosis research program in Sao Paulo, Brazil. Organization and implementation. Clin Neuropsychiatry J Treat Eval. 2008;5:273-8.

22. Schultze-Lutter F, Ruhrmann S, Klosterkötter J. Early detection of psychosisestablishing a service for persons at risk. Eur Psychiatry. 2009;24:1-10.

23. Janssen $H$, Maat A, Slot MIE, Scheepers F. Efficacy of psychological interventions in young individuals at ultra-high risk for psychosis: a naturalistic study. Early Interv Psychiatry. 2020;15:1019-27.

24. Kotlicka-Antczak M, Pawełczyk T, Rabe-Jabłońska J, Pawełczyk A. PORT (Programme of Recognition and Therapy): the first Polish recognition and treatment programme for patients with an at-risk mental state. Early Inter Psychiatry. 2015;9:339-42.

25. Kotlicka-Antczak M, Pawełczyk T, Podgórski M, Żurner N, Karbownik MS, Pawełczyk A. Polish individuals with an at-risk mental state: demographic and clinical characteristics. Early Inter Psychiatry. 2016;12:391-9.

26. Kwon JS, Byun MS, Lee TY, An SK. Early intervention in psychosis: insights from Korea. Asian J Psychiatr. 2012;5:98-105.

27. Rao S, Pariyasami S, Tay S, Lim L, Yuen S, Poon L. et al. Support for Wellness Achievement Programme (SWAP): a service for individuals with at-risk mental state in Singapore. Ann Acad Med Singap. 2013;42:552-5.

28. Tay SA, Yuen S, Lim LK, Pariyasami S, Rao S, Poon LY, et al. Support for Wellness Achievement Programme (SWAP): clinical and demographic characteristics of young people with at-risk mental state in Singapore. Early Inter Psychiatry. 2015;9:516-22.

29. Broome MR, Woolley JB, Johns LC, Valmaggia LR, Tabraham P, Gafoor R, et al. Outreach and support in south London (OASIS): implementation of a clinical service for prodromal psychosis and the at risk mental state. Eur Psychiatry. 2005;20:372-8.

30. Fusar-Poli $P$, Byrne $M$, Badger S, Valmaggia LR, McGuire PK. Outreach and support in South London (OASIS), 2001-2011: ten years of early diagnosis and treatment for young individuals at high clinical risk for psychosis. Eur Psychiatry. 2013;28:315-26.

31. Fusar-Poli $P$, Frascarelli $M$, Valmaggia $L$, Byrne $M$, Stahl $D$, Rocchetti $M$, et al. Antidepressant, antipsychotic and psychological interventions in subjects at high clinical risk for psychosis: OASIS 6-year naturalistic study. Psychol Med. 2014;45:1327-39.

32. Fusar-Poli P, Spencer T, De Micheli A, Curzi V, Nandha S, McGuire P. Outreach and support in South-London (OASIS) 2001-2020: twenty years of early detection, prognosis and preventive care for young people at risk of psychosis. Eur Neuropsychopharmacol. 2020;39:111-22. 
33. Green CEL, McGuire PK, Ashworth M, Valmaggia LR. Outreach and Support in South London (OASIS). Outcomes of non-attenders to a service for people at high risk of psychosis: the case for a more assertive approach to assessment. Psychol Med. 2011;41:243-50.

34. Tognin S, Grady L, Ventura S, Valmaggia L, Sear V, McGuire P, et al. The provision of Education and employment support at the outreach and support in South London (OASIS) service for people at clinical high risk for psychosis. Front Psychiatry. 2019;10:1-9.

35. Power $P$, McGuire $P$, lacoponi E, Garety $P$, Morris E, Valmaggia L, et al. Lambeth Early Onset (LEO) and Outreach \& Support in South London (OASIS) service. Early Inter Psychiatry. 2007;1:97-103.

36. Fusar-Poli P, Estradé A, Spencer TJ, Gupta S, Murguia-Asensio S, Eranti S, et al. PanLondon Network for Psychosis-Prevention (PNP). Front Psychiatry. 2019;10:1-10.

37. McFarlane WR, Cook WL, Downing D, Verdi MB, Woodberry KA, Ruff A. Portland identification and early referral: a community-based system for identifying and treating youths at high risk of psychosis. Psychiatr Serv. 2010;61:512-5.

38. Ruff A, McFarlane WR, Downing D, Cook W, Woodberry K. A community outreach and education model for early identification of mental illness in young people. Adolesc Psychiatry. 2012;2:140-5.

39. Coates D, Wright L, Moore T, Pinnell S, Merillo C, Howe D. The psychiatric, psychosocial and physical health profile of young people with early psychosis: data from an early psychosis intervention service. Child Youth Serv. 2019;40:93-115.

40. Carr V, Halpin S, Lau N, O'brien S, Beckmann J, Lewin T. A risk factor screening and assessment protocol for schizophrenia and related psychosis. Aust N. Z J Psychiatry. 2000;34:S170-S180.

41. Penno SJ, Hamilton B, Petrakis M. Early intervention in psychosis: health of the Nation Outcome Scales (HoNOS) outcomes from a five-year prospective study. Arch Psychiatr Nurs. 2017;31:553-60.

42. Selvendra A, Baetens D, Trauer T, Petrakis M, Castle D. First episode psychosis in an adult area mental health service-a closer look at early and late-onset first episode psychosis. Australas Psychiatry. 2014;22:235-41.

43. Pruessner M, Faridi K, Shah J, Rabinovitch M, lyer S, Abadi S, et al. The Clinic for Assessment of Youth at Risk (CAYR): 10 years of service delivery and research targeting the prevention of psychosis in Montreal, Canada. Early Inter Psychiatry. 2015;11:177-84.

44. Addington J, Epstein I, Reynolds A, Furimsky I, Rudy L, Mancini B, et al. Early detection of psychosis: finding those at clinical high risk. Early Inter Psychiatry. 2008;2:147-53.

45. Gaspar PA, Castillo RI, Maturana A, Villar MJ, Ulloa K, González G, et al. Early psychosis detection program in Chile: a first step for the South American challenge in psychosis research. Early Inter Psychiatry. 2018;13:328-34.

46. Wong GHY, Hui CLM, Tang JYM, Chang WC, Chan SKW, Xu JQ, et al. Early intervention for psychotic disorders: real-life implementation in Hong Kong. Asian J Psychiatr. 2012;5:68-72.

47. Wong GH, Hui $\mathrm{CL}$, Chiu CP, Lam M, Chung DW, Tso S, et al. Early detection and intervention for psychosis in Hong Kong: experience of a population-based intervention programme. Clin Neuropsychiatry J Treat Eval. 2008;5:286-9.

48. Tang JYM, Wong GHY, Hui CLM, Lam MML, Chiu CPY, Chan SKW, et al. Early intervention for psychosis in Hong Kong - the EASY programme. Early Inter Psychiatry. 2010;4:214-9.

49. Oppetit A, Bourgin J, Martinez G, Kazes M, Mam-Lam-Fook C, Gaillard R, et al. The C'JAAD: a French team for early intervention in psychosis in Paris. Early Inter Psychiatry. 2016;12:243-9.

50. Kollias C, Xenaki LA, Dimitrakopoulos S, Kosteletos I, Kontaxakis V, Stefanis N, et al. Early psychosis intervention outpatient service of the 1st psychiatric university clinic in Athens: 3 years of experience. Early Inter Psychiatry. 2016:12:491-6.

51. Kollias K, Xenaki L, Vlachos I, Dimitrakopoulos S, Kosteletos I, Nianiakas N, et al. The development of the Early Intervention in Psychosis (EIP) outpatient unit of Eginition University Hospital into an EIP Network. Psychiatriki. 2020;31:177-82.

52. Cocchi A, Balbi A, Corlito G, Ditta G, Di Munzio W, Nicotera M, et al. Early intervention in psychosis: a feasibility study financed by the Italian Center on Control of Maladies. Early Inter Psychiatry. 2015;9:163-71.

53. Parabiaghi A, Confalonieri L, Magnani N, Lora A, Butteri E, Prato K, et al. Integrated programs for early recognition of severe mental disorders: recommendations from an Italian multicenter project. Front Psychiatry. 2019;10:1-6.

54. Belvederi Murri M, Bertelli R, Carozza P, Berardi L, Cantarelli L, Croce E, et al. Firstepisode psychosis in the Ferrara Mental Health Department: incidence and clinical course within the first 2 years. Early Interv Psychiatry. 2020;15:1738-48.

55. Leuci E, Quattrone E, Pellegrini P, Pelizza L. The "Parma-Early Psychosis" program: general description and process analysis after 5 years of clinical activity. Early Interv Psychiatry. 2019;14:356-64.

56. Cocchi A, Meneghelli A, Erlicher A, Pisano A, Cascio MT, Preti A. Patterns of referral in first-episode schizophrenia and ultra high-risk individuals: results from an early intervention program in Italy. Soc Psychiatry Psychiatr Epidemiol. 2013:48:1905-16.

57. Meneghelli A, Cocchi A, Preti A. 'Programma2000': a multi-modal pilot programme on early intervention in psychosis underway in Italy since 1999 . Early Inter Psychiatry. 2010;4:97-103.

58. Meneghelli A, Barbera S, Meliante M, Monzani E, Preti A, Cocchi A, et al. Outcome at 2-year of treatment in first-episode psychosis patients who were enrolled in a specialized early intervention program. Psychiatry Res. 2020;291:113200.

59. Pelizza L, Azzali S, Paterlini F, Garlassi S, Scazza I, Chiri LR, et al. The "Reggio Emilia At-Risk Mental States" program: a diffused, "liquid" model of early intervention in psychosis implemented in an Italian Department of Mental Health. Early Inter Psychiatry. 2019;13:1513-24.

60. Pelizza L, Azzali S, Garlassi S, Scazza I, Paterlini F, Chiri LR, et al. Assessing aberrant salience in young community help-seekers with early psychosis: the approved Italian version of the Aberrant Salience Inventory. J Clin Psychol 2020. https://doi.org/10.1002/jclp.23059.

61. Poletti M, Pelizza L, Azzali S, Paterlini F, Garlassi S, Scazza I, et al. Overcoming the gap between child and adult mental health services: the Reggio Emilia experience in an early intervention in psychosis program. Early Interv Psychiatry. 2020;15:1749-58.

62. Katsura M, Ohmuro N, Obara C, Kikuchi T, Ito F, Miyakoshi T, et al. A naturalistic longitudinal study of at-risk mental state with a 2.4 year follow-up at a specialized clinic setting in Japan. Schizophr Res. 2014;158:32-38.

63. Joa I, Bjornestad J, Johannessen JO, Langeveld J, Stain HJ, Weibell M, et al. Early detection of ultra high risk for psychosis in a Norwegian catchment area: the two year follow-up of the prevention of psychosis study. Front Psychiatry. 2021;12:1-10.

64. Joa I, Gisselgård J, Brønnick K, McGlashan T, Johannessen JO. Primary prevention of psychosis through interventions in the symptomatic prodromal phase, a pragmatic Norwegian ultra high risk study. BMC Psychiatry. 2015;15:1-9.

65. Kim SW, Kim JK, Jhon M, Lee HJ, Kim H, Kim JW, et al. Mindlink: a stigma-free youth-friendly community-based early-intervention centre in Korea. Early Interv Psychiatry. 2020;15:1389-94.

66. Quijada Y, Tizón JL, Artigue J, Parra B. At-risk mental state (ARMS) detection in a community service center for early attention to psychosis in Barcelona. Early Inter Psychiatry. 2010;4:257-62.

67. Ortega L, Montalvo I, Solé $M$, Creus M, Cabezas Á, Gutiérrez-Zotes A, et al. Relationship between childhood trauma and social adaptation in a sample of young people attending an early intervention service for psychosis. Rev Psiquiatr Salud Ment. 2020;13:131-9.

68. Theodoridou A, Heekeren K, Dvorsky D, Metzler S, Franscini M, Haker H, et al. Early recognition of high risk of bipolar disorder and psychosis: an overview of the ZInEP 'early recognition' study. Front Public Heal. 2014;2:1-8.

69. Riecher-Rössler A, Gschwandtner U, Aston J, Borgwardt S, Drewe M, Fuhr P, et al. The Basel early-detection-of-psychosis (FEPSY)-study-design and preliminary results. Acta Psychiatr Scand. 2007;115:114-25.

70. Simon AE, Theodoridou A, Schimmelmann B, Schneider R, Conus P. The Swiss Early Psychosis Project SWEPP: a national network. Early Inter Psychiatry. 2012;6:106-11.

71. Michel $C$, Kaess M, Flückiger R, Büetiger JR, Schultze-Lutter F, Schimmelmann BG, et al. The Bern Early Recognition and Intervention Centre for mental crisis (FETZ Bern)—An 8-year evaluation. Early Interv Psychiatry 2021. https://doi.org/ 10.1111/eip.13160.

72. Ventura J, Jouini L, Aissa A, Larnaout A, Nefzi R, Ghazzai M, et al. Establishing a clinical high-risk program in Tunisia, North Africa: a pilot study in early detection and identification. Early Interv Psychiatry. 2021;5:1777-83.

73. Adamson V, Barrass $\mathrm{E}$, McConville S, Irikok C, Taylor K, Pitt S, et al. Implementing the access and waiting time standard for early intervention in psychosis in the United Kingdom: an evaluation of referrals and post-assessment outcomes over the first year of operation. Early Inter Psychiatry. 2018;12:979-86.

74. Tiffin PA, Hudson S. Early intervention in the real world: an early intervention in psychosis service for adolescents. Early Inter Psychiatry. 2007;1:212-8.

75. Lynch S, McFarlane WR, Joly B, Adelsheim S, Auther A, Cornblatt BA, et al. Early detection, intervention and prevention of psychosis program: community outreach and early identification at six U.S. sites. Psychiatr Serv. 2016;67:510-6.

76. McFarlane WR, Cook WL, Downing D, Ruff A, Lynch S, Adelsheim S, et al. Early detection, intervention, and prevention of psychosis program: rationale, design, and sample description. Adolesc Psychiatry. 2012;2:112-24.

77. Bertulies-Esposito B, Nolin M, lyer SN, Malla A, Tibbo $P$, Otter $N$, et al. Où en sommes-nous? An overview of successes and challenges after 30 years of early intervention services for psychosis in Quebec. Can J Psychiatry 2020. https://doi. org/10.1177/0706743719895193.

78. Cocchi A, Cavicchini A, Collavo M, Ghio L, Macchi S, Meneghelli A, et al. Implementation and development of early intervention in psychosis services in Italy: a national survey promoted by the Associazione Italiana Interventi Precoci nelle Psicosi. Early Inter Psychiatry. 2015;12:37-44. 
79. Ghio L, Natta W, Peruzzo L, Gotelli S, Tibaldi G, Ferrannini L. Process of implementation and development of early psychosis clinical services in Italy: a survey. Early Inter Psychiatry. 2012;6:341-6.

80. Coentre R, Levy P. Early intervention in psychosis: The first national survey in Portugal. Schizophr Res. 2020;218:298-9.

81. Stain HJ, Mawn L, Common S, Pilton M, Thompson A. Research and practice for ultra-high risk for psychosis: a national survey of early intervention in psychosis services in England. Early Interv Psychiatry. 2017;13:47-52.

82. UN General Assembly. Transforming our world: the 2030 agenda for sustainable development. United Nations: New York;2015.

83. Vogel DL, Wade NG, Hackler AH. Perceived public stigma and the willingness to seek counseling: the mediating roles of self-stigma and attitudes toward counseling. J Couns Psychol. 2007;54:40-50.

84. Rüsch N, Zlati A, Black G, Thornicroft G. Does the stigma of mental illness contribute to suicidality? Br J Psychiatry. 2014;205:257-9.

85. Salazar de Pablo G, De Micheli A, Nieman DH, Correll CU, Kessing LV, Pfennig A, et al. Universal and selective interventions to promote good mental health in young people: systematic review and meta-analysis. Eur Neuropsychopharmacol. 2020;41:28-39.

86. Thornicroft G, Mehta N, Clement S, Evans-Lacko S, Doherty M, Rose D, et al. Evidence for effective interventions to reduce mental-health-related stigma and discrimination. Lancet. 2016;387:1123-32.

87. Teasdale SB, Burrows TL, Hayes T, Hsia CY, Watkins A, Curtis J, et al. Dietary intake, food addiction and nutrition knowledge in young people with mental illness. Nutr Diet. 2019;77:315-22.

88. Carney R, Bradshaw T, Yung AR. Monitoring of physical health in services for young people at ultra-high risk of psychosis. Early Inter Psychiatry. 2018;12:153-9.

89. Firth J, Siddiqi N, Koyanagi A, Siskind D, Rosenbaum S, Galletly C, et al. The Lancet Psychiatry Commission: a blueprint for protecting physical health in people with mental illness. Lancet Psychiatry. 2019;6:675-712.

90. Gore FM, Bloem PJN, Patton GC, Ferguson J, Joseph V, Coffey C, et al. Global burden of disease in young people aged 10-24S years: a systematic analysis. Lancet. 2011;377:2093-102.

91. Jacka FN, Mykletun A, Berk M. Moving towards a population health approach to the primary prevention of common mental disorders. BMC Med 2012. https:// doi.org/10.1186/1741-7015-10-149.

92. Firth J, Solmi M, Wootton RE, Vancampfort D, Schuch FB, Hoare E, et al. A metareview of "lifestyle psychiatry": the role of exercise, smoking, diet and sleep in the prevention and treatment of mental disorders. World Psychiatry. 2020;19:360-80.

93. O'Neil A, Jacka FN, Quirk SE, Cocker F, Taylor CB, Oldenburg B, et al. A shared framework for the common mental disorders and non-communicable disease: key considerations for disease prevention and control. BMC Psychiatry. 2015;15:1-6.

94. Guloksuz S, Pries LK, ten Have M, de Graaf R, van Dorsselaer S, Klingenberg B, et al. Association of preceding psychosis risk states and non-psychotic mental disorders with incidence of clinical psychosis in the general population: a prospective study in the NEMESIS-2 cohort. World Psychiatry. 2020;19:199-205.

95. Ajnakina O, David AS, Murray RM. 'At risk mental state' clinics for psychosis-An idea whose time has come-And gone! Psychol Med. 2019;49:529-34.

96. Murray RM, David AS, Ajnakina O. Prevention of psychosis: moving on from the at-risk mental state to universal primary prevention. Psychol Med. 2021;51:223-7.

97. McGorry P, Trethowan J, Rickwood D. Creating headspace for integrated youth mental health care. World Psychiatry. 2019;18:140-1.

98. Fusar-Poli $P$. Integrated mental health services for the developmental period $(0$ to 25 years): a critical review of the evidence. Front Psychiatry. 2019;10:1-17.

99. Domingues I, Alderman T, Cadenhead KS. Strategies for effective recruitment of individuals at risk for developing psychosis. Early Inter Psychiatry. 2011;5:233-41.

100. Salazar De Pablo G, De Micheli A, Solmi M, Oliver D, Catalan A, Verdino V, et al. Universal and selective interventions to prevent poor mental health outcomes in young people: systematic review and meta-analysis. Harv Rev Psychiatry. 2021;29:196-215.

101. Brietzke E, Araripe Neto AG, Dias Á, Mansur RB, Bressan RA. Early intervention in psychosis: a map of clinical and research initiatives in Latin America. Rev Bras Psiquiatr. 2011;33:s213-24.
102. Aceituno D, Mena C, Vera N, Gonzalez-Valderrama A, Gadelha A, Diniz E, et al. Implementation of early psychosis services in Latin America: a scoping review. Early Inter Psychiatry. 2021;15:1104-14.

\section{ACKNOWLEDGEMENTS}

We thank the team at the OASIS UK service for the mental health literacy promotion materials included in eFig. 2. Materials are authored by Jennifer Harries, a member of the OASIS team.

\section{AUTHOR CONTRIBUTIONS}

$A E$ contributed to the design, literature review data extraction, and writing. GSdP and AZ contributed to the literature review and data extraction. SW and HLF contributed to reading, reviewing and editing. PF-P contributed to design, supervision, reading, reviewing and editing.

\section{FUNDING}

This study is supported by a Wellcome Trust grant $(215793 / Z / 19 / Z)$ to PF-P. PF-P is supported by the National Institute for Health Research (NIHR) Mental Health Biomedical Research Centre at South London and Maudsley NHS Foundation Trust and King's College London. HLF receives salary support from the Economic and Social Research Council (ESRC) Centre for Society and Mental Health at King's College London [ES/S012567/1]. The views expressed are those of the authors and not necessarily those of the Wellcome Trust, National Health Service, the NIHR, the Department of Health and Social Care, the ESRC, or King's College London.

\section{COMPETING INTERESTS}

The authors declare no competing interests.

\section{ADDITIONAL INFORMATION}

Supplementary information The online version contains supplementary material available at https://doi.org/10.1038/s41398-022-01805-4.

Correspondence and requests for materials should be addressed to Paolo Fusar-Poli.

Reprints and permission information is available at http://www.nature.com/ reprints

Publisher's note Springer Nature remains neutral with regard to jurisdictional claims in published maps and institutional affiliations.

\begin{abstract}
adaptation, distribution and reproduction in any medium or format, as long as you give appropriate credit to the original author(s) and the source, provide a link to the Creative Commons license, and indicate if changes were made. The images or other third party material in this article are included in the article's Creative Commons license, unless indicated otherwise in a credit line to the material. If material is not included in the article's Creative Commons license and your intended use is not permitted by statutory regulation or exceeds the permitted use, you will need to obtain permission directly from the copyright holder. To view a copy of this license, visit http://creativecommons. org/licenses/by/4.0/.
\end{abstract}

Open Access This article is licensed under a Creative Commons Attribution 4.0 International License, which permits use, sharing,

(c) The Author(s) 2022 